\title{
Duration of Influenza Virus Shedding Among HIV-Infected Adults in the cART Era, 2010-2011
}

\author{
Pragna Patel, Timothy Bush,, E. Milu Kojic,2 Edgar T. Overton, ${ }^{3}$ Keith Henry, Nur Önen, \\ Frank Rhame, Lois Conley, John T. Brooks, and Alicia Fry ${ }^{7}$
}

\begin{abstract}
The duration of influenza virus shedding in HIV-infected adults is unknown and could affect quarantine and treatment recommendations. Participants were monitored for influenza-like illness (ILI), defined as fever and cough or sore throat, using weekly telephone audio computer-assisted self-interviews. Those with ILI were further evaluated at three HIV specialty clinics. For those with influenza, we collected nasopharyngeal washes every 3 days after the date of confirmed influenza infection for 21-28 days; specimens underwent reverse transcriptase - polymerase chain reaction (RT-PCR) and viral culture. Duration of influenza virus shedding was the interval from the date of onset (day 0) of ILI to the date of last culture-positive specimen. Characteristics were compared between patients with and without influenza using Fisher's exact test. We used the Wilcoxon rank-sum test to examine factors that may have affected influenza virus shedding. From October 2010 to April 2011, we enrolled 961 participants in syndromic surveillance and diagnosed 20 patients with influenza whose characteristics were as follows: median age 48 years (interquartile range [IQR]: 43-53), $60 \%$ male, 50\% nonHispanic black, 95\% had been prescribed combination highly active antiretroviral therapy (cART), $85 \%$ were virologically suppressed (HIV RNA <400 copies/ml), median CD4 cell count 317 cells $/ \mathrm{mm}^{3}$ (IQR: 190-544), and median follow-up time 21 days (IQR: 19-22). Compared with persons without influenza, persons with influenza were more likely to be older, use injection drugs, and have a lower median CD4 cell count and were less likely to have had an influenza vaccination in the past 12 months. Median durations of shedding, PCR detection, and ILI symptoms were 3 (IQR: 0-5), 10 (IQR: 6-15), and 14 days (IQR: 12-26), respectively. Median days of shedding were similar among patients with and without any prior influenza vaccination $(0$ vs. 4 , $p=.448$ ), HIV viral suppression ( 2 vs. $6, p=.053$ ), and oseltamivir use ( 5 vs. $0, p=.083$ ). HIV-infected persons on cART in our study shed influenza virus for a similar duration as that reported for HIV-uninfected persons.
\end{abstract}

Keywords: HIV, influenza, shedding

\section{Introduction}

N HEALTHY INDIVIDUALS, influenza is usually a self-limited illness; affected persons rapidly mount protective antibody responses to the viral infection and may shed virus for up to $7-$ 10 days. $^{1-5}$ Influenza virus infection is a common cause of respiratory illness among HIV-infected persons ${ }^{6}$ for whom in- fluenza can be more severe and more prolonged and is associated with greater attributable mortality among persons with AIDS $^{7-12}$ Several studies have described prolonged shedding of influenza virus (from over 2 weeks to as long as 18 months) in patients with congenital immunodeficiency or who have been immunocompromised by cancer and its treatment, including bone marrow transplants. ${ }^{13-20}$ Among such immunosuppressed

\footnotetext{
${ }^{1}$ Division of HIV/AIDS Prevention, Centers for Disease Control and Prevention, Atlanta, Georgia.

${ }^{2}$ Department of Infectious Diseases, Brown University, Providence, Rhode Island.

${ }^{3}$ University of Alabama at Birmingham School of Medicine, Birmingham, Alabama.

${ }^{4}$ Hennepin County Medical Center, University of Minnesota, Minneapolis, Minnesota.

${ }^{5}$ Washington University School of Medicine, St. Louis, Missouri.

${ }^{6}$ Abbott-Northwestern Hospital, Minneapolis, Minnesota.

${ }^{7}$ Influenza Division, Centers for Disease Control and Prevention, Atlanta, Georgia.
}

(C) Pragna Patel, et al., 2016; Published by Mary Ann Liebert, Inc. This Open Access article is distributed under the terms of the Creative Commons Attribution Noncommercial License (http://creativecommons.org/licenses/by-nc/4.0/) which permits any noncommercial use, distribution, and reproduction in any medium, provided the original author(s) and the source are credited. 
patients, the influenza virus is more likely to develop resistance to antiviral drugs ${ }^{13-20}$ and studies have shown that resistant influenza virus can be effectively transmitted. ${ }^{21,22}$

Data on influenza virus shedding dynamics and the risk for developing antiviral resistance have not been described for persons living with HIV infection, who comprise an estimated 1.2 million persons in the United States. ${ }^{23}$ If HIVinfected patients responded to influenza virus infection in a manner similar to patients immunocompromised by other conditions, they could not only substantially amplify influenza transmission but also add to selection pressure favoring the emergence of resistant strains of the virus. Understanding whether HIV-infected persons experience prolonged shedding of influenza virus and are predisposed to developing antiviral resistance has important public health implications, especially regarding quarantine and treatment recommendations. Yet, there are inadequate data from HIV-infected persons to inform such recommendations. As a result, findings from studies of otherwise immunocompromised persons have been extrapolated to HIV-infected persons. However, the qualitatively different nature of conditions such as cancer and its treatment compared with HIV infection limits, if not precludes, this generalization.

To inform whether quarantine and treatment recommendations for influenza among HIV-infected persons should differ from recommendations made for otherwise immunocompetent persons, we conducted a study of influenza shedding among HIV-infected adults. We aimed to describe the duration of influenza virus shedding and to correlate duration of influenza virus shedding with influenza signs and symptoms, CD4 cell count, and HIV viral load. Furthermore, we assessed factors that may affect influenza virus shedding dynamics, including antiviral treatment and influenza vaccination.

\section{Materials and Methods}

\section{Study population}

The Study to Understand the Natural History of HIV/AIDS in the Era of Effective Therapy (the SUN Study). The SUN Study was a CDC-funded closed prospective cohort study that enrolled patients receiving routine outpatient care at seven HIV specialty clinics in four U.S. cities. Enrollment began March 2004 and closed June 2006 with 700 participants; data collection ceased on June 30, 2012. Patients were eligible for enrollment if they were antiretroviral naive or their prior antiretroviral exposure had consisted solely of combination highly active antiretroviral therapy (cART) with no more than 30 days of cumulative exposure to any mono- or dualantiretroviral regimen (e.g., treatment to prevent mother-tochild transmission of HIV). Details about the SUN Study have been published elsewhere. ${ }^{24}$ SUN study participants were eligible for enrollment into the influenza study, but because SUN patients were relatively healthy, sites were asked to also enroll non-SUN HIV-infected patients who may have had lower CD4 cell counts. The study protocol was reviewed and approved by the institutional review boards of the Centers for Disease Control and Prevention and the participating clinical centers. All participants provided written informed consent.

\section{Study design}

Phase I-Syndromic surveillance. From October 1, 2010 to April 30, 2011, participating SUN Study HIV specialty clinics were asked to enroll all of their HIV-infected patients in syndromic surveillance for influenza. For patients who agreed to enroll in syndromic surveillance, we collected baseline sociodemographic data as well as information about underlying conditions that could have increased the risk for influenza infection or severe influenza illness, such as prior influenza vaccination history and history of smoking. These data were used to compare persons with and without influenza at study completion. Participants were monitored for influenza-like illness (ILI), defined as fever and cough or sore throat, using weekly telephone audio computer-assisted self-interviews. Participants were called using a computerized system and asked to complete, using their telephone, a short survey about ILI symptoms experienced within the preceding week. Participants who met the case definition for ILI based on survey responses were called by the study nurse and asked to come to the clinic for further evaluation. The telephone survey also reminded participants to seek care as soon as they developed ILI, at which time they were assessed for potential enrollment into the influenza shedding study (see below). Participants with possible influenza were quarantined away from other patients during clinical evaluation to reduce potential transmission of influenza. Nasopharyngeal (NP) washes were collected from persons with ILI and assayed for influenza infection by reverse transcriptase (RT) PCR at local laboratories that served the participating HIV specialty clinics. All patients with ILI were clinically assessed and offered antiviral therapy, if indicated, pending reverse transcriptase - polymerase chain reaction (RTPCR) confirmation of infection.

Phase II-Influenza shedding study. Participants with confirmed influenza infection were asked to enroll in the second phase of the study to determine the duration of influenza virus shedding. Consenting participants had serial follow-up visits to collect NP washes and other specimens, as detailed below. To correlate influenza virus shedding with symptoms and to determine the baseline date of ILI onset, a detailed symptom history was collected at enrollment into the shedding study and at each subsequent study visit when specimens were collected. These questionnaires also collected information regarding use of prescribed influenza antiviral medications. To assess factors that may have affected influenza shedding and to describe how duration of influenza virus shedding was affected by antiviral treatment, patient's consent was obtained to permit abstraction from his/her medical record for diagnoses that might have rendered the patient at higher risk for influenza infection, use of antiretroviral treatments (including duration), history of prior vaccinations, including influenza and pneumococcal vaccination, and details regarding any treatments (e.g., prescriptions of antiviral agents) and complications attributable to influenza (e.g., hospitalizations, deaths) while enrolled in the study. Participant self-report and medical record information was reconciled through adjudication by the local site investigator and the participant's care provider.

\section{Specimen collection and testing}

NP washes were performed with the patient seated and their head in a semi-Fowler (sniffing) position, holding a sterile cup under their nose. With the patient leaning forward, occluding one nostril, and holding his/her breath, saline was pushed into the open nostril. The wash was collected in the 
sterile cup. The wash was drawn from the cup into a syringe (recover 1-2 cc) and squirted into cryotube and placed on ice. NP specimens were batched and stored at $-70^{\circ} \mathrm{C}$.

We collected NP washes every 3 days for 21 days following the date of influenza confirmation. We allowed a 1day window period on either side of the scheduled study visit date to accommodate weekends, holidays, emergencies, and missed visits. If a participant continued to experience symptoms at the day-21 visit, we collected an additional final set of NP washes at day 28. All NP wash specimens, including the initial screening specimens, were split into at least two aliquots and stored at $-70^{\circ} \mathrm{C}$ until a later time when they were run in batches sequentially for RT-PCR and viral culture at a single central laboratory. All specimens underwent RT-PCR for influenza A and B, with subtyping for influenza A-positive specimens using a laboratory-developed test. RT-PCR cycle threshold $(\mathrm{Ct})$ values were recorded and assumed to be a correlate for influenza viral RNA load values. Influenza virus type and subtype were recorded. If an NP wash was positive for influenza by RT-PCR, an aliquot was cultured for virus isolation. Virus was isolated using the CDC protocol for virus propagation in MDCK cells and RMK cells using traditional cell culture tubes as well as by R-Mix culture, which is a commercial system that consists of centrifuging the specimen onto a cell monolayer containing a mixture of cells optimized for respiratory virus recovery and then staining the monolayer with monoclonal antibodies specific for viral antigens. Three wells were inoculated and then read at days 1,2 , and 3. Specimens that were positive by $>2$ methods were considered positive. Resistance testing was performed using sequencing using $\mathrm{CDC} \mathrm{PCR} /$ sequencing primers for amplifying and sequencing fragments $\mathrm{A}, \mathrm{B}$, and $\mathrm{C}$ of the $\mathrm{N}$ gene. PCR and resistance testing were performed at the Special Project's Laboratory at Washington University. Viral culture was performed at the Clinical Virology Laboratory at St Louis Children's Hospital.

To assess the effect of influenza on immune status and on control of HIV infection, serum specimens were drawn for CBC and CD4 cell count at enrollment and at the patient's next HIV clinic visit at least 60 days after and closest to 90 days after the onset date of ILI (two samples total). Plasma specimens were drawn for HIV viral load quantification at the enrollment visit and subsequent study visits at day 9 and day 21 and at the patient's next HIV clinic at least 60 days after and closest to 90 days after the onset date of ILI (four samples total) (Table 1). CBC, CD4 cell count, and HIV viral load quantification were performed at local laboratories that served each participating HIV specialty clinic.

\section{Statistical analyses}

Continuous variables were summarized as medians with interquartile ranges (IQRs), and percentages for each category of categorical variables were calculated. Characteristics were compared between patients with and without influenza using Fisher's exact test. We considered $p$ values less than .05 statistically significant. We defined the duration of influenza virus shedding as the interval from date of ILI onset to the date of last culture-positive NP wash. This definition of shedding duration was used for all analyses. However, to correlate symptoms with shedding, we defined the duration of influenza virus shedding as the interval from date of ILI onset to the date of last PCR-positive NP wash, as PCR is the screening test for influenza, and thus, most clinically relevant in this context. We used the Wilcoxon rank-sum test to examine factors that may have affected influenza virus shedding. ${ }^{25}$ We calculated correlation coefficients to assess the linear association between variables. All analyses were conducted in SAS 9.2 (SAS Institute, Cary, NC).

\section{Results}

\section{Patient characteristics}

From October 2010 to April 2011, we enrolled 961 participants in syndromic surveillance and diagnosed 20 patients with PCR-confirmed influenza infection; all 20 agreed to enroll in the shedding study. Compared with persons without influenza, persons with influenza were more likely to be older, use injection drugs, have a lower median CD4 cell count, and were less likely to have had an influenza vaccination in the past 12 months (Table 2). For the 20 patients with influenza, demographic and clinical characteristics were as follows: median age 48 years (IQR 43-53), $60 \%$ male, $50 \%$ non-Hispanic black, $44 \%$ current smokers, $95 \%$ prescribed HAART, $85 \%$ virologically suppressed (HIV RNA $<400$ copies/ml), median CD4 cell count 317 cells $/ \mathrm{mm}^{3}$ (IQR: 190-544), and median follow-up time after confirmation of influenza infection 21 days (IQR: 19-22). The most common symptoms reported were cough $(100 \%)$, fever $(90 \%)$, myalgias $(80 \%)$, sore throat $(75 \%)$, and headache

Table 1. Schedule for Laboratory Evaluations for Influenza Shedding Study (Phase II)

\begin{tabular}{|c|c|c|c|c|c|c|c|c|c|c|}
\hline \multirow[b]{5}{*}{ Laboratory evaluation } & & \multicolumn{8}{|c|}{ Postentry evaluations (days) } & \multirow[b]{3}{*}{ Next clinic visit $(\sim 90$ days $)$} \\
\hline & \multirow[b]{2}{*}{ Date of flu Dx (Day 0) } & Entry-3 & 6 & 9 & 12 & 15 & 18 & 21 & 28 & \\
\hline & & \multicolumn{8}{|c|}{ \pm 1 days } & \\
\hline & \multicolumn{10}{|c|}{ Visit number } \\
\hline & 00 & 01 & 02 & 03 & 04 & 05 & 06 & 07 & 08 & 09 \\
\hline $\mathrm{CBC}$ & & $\mathrm{X}$ & & & & & & & & $\mathrm{X}$ \\
\hline CD4+/CD8+ & & $\mathrm{X}$ & & & & & & & & $\mathrm{X}$ \\
\hline Plasma HIV-1 RNA & & $\mathrm{X}$ & & $\mathrm{X}$ & & & & $\mathrm{X}$ & & $\mathrm{X}$ \\
\hline $\begin{array}{l}\text { Nasopharyngeal wash } \\
\text { for influenza } \\
\text { culture and PCR }\end{array}$ & $X$ & $\mathrm{X}$ & $X$ & $\mathrm{X}$ & $\mathrm{X}$ & $\mathrm{X}$ & $\mathrm{X}$ & $\mathrm{X}$ & $\mathrm{X}$ & \\
\hline
\end{tabular}


Table 2. Characteristics of Study Participants Without Influenza Compared With Participants Diagnosed with Influenza, 2010-2011 $(N=941)$

\begin{tabular}{|c|c|c|c|c|}
\hline Characteristic & $\begin{array}{l}\text { All participants } \\
\qquad(\mathrm{n}=961)\end{array}$ & $\begin{array}{l}\text { No influenza } \\
\quad(\mathrm{n}=941)\end{array}$ & $\begin{array}{c}\text { Influenza } \\
(\mathrm{n}=20)\end{array}$ & $\mathrm{p}$ \\
\hline Median age, years (IQR) & $45(35-51)$ & $45(35-51)$ & $48(43-53)$ & .045 \\
\hline Male gender, $n(\%)$ & $666(69)$ & $654(70)$ & $12(60)$ & .339 \\
\hline \multicolumn{5}{|l|}{ Race/ethnicity, $n(\%)$} \\
\hline White & $379(40)$ & $373(40)$ & $6(30)$ & \multirow[t]{4}{*}{$.060^{\mathrm{a}}$} \\
\hline Black & $485(51)$ & $475(51)$ & $10(50)$ & \\
\hline Hispanic & $73(8)$ & $69(7)$ & $4(20)$ & \\
\hline Other & $15(2)$ & $15(2)$ & $0(0)$ & \\
\hline \multicolumn{5}{|l|}{ Mode of HIV transmission, $n(\%)$} \\
\hline MSM & $457(48)$ & $451(49)$ & $6(30)$ & \multirow[t]{5}{*}{$.020^{b}$} \\
\hline IDU & $109(11)$ & $103(11)$ & $6(30)$ & \\
\hline Heterosexual & $293(31)$ & $286(31)$ & $7(35)$ & \\
\hline Other & $17(2)$ & $17(2)$ & $0(0)$ & \\
\hline Unknown & $72(8)$ & $71(8)$ & $1(5)$ & \\
\hline Current smoking, $n(\%)$ & $448(49)$ & $441(49)$ & $7(44)^{c}$ & .803 \\
\hline Median CD4 cell count (cells $\left./ \mathrm{mm}^{3}\right)(\mathrm{IQR})$ & $480(313-680)$ & $484(314-683)$ & $317(190-544)$ & .014 \\
\hline CD 4 cell count $<200$ cells $/ \mathrm{mm}^{3}, n(\%)$ & $120(13)$ & $115(12)$ & $5(25)$ & .092 \\
\hline HIV RNA <400 copies/ml & $748(79)$ & $731(79)$ & $17(85)$ & .593 \\
\hline Influenza vaccination in past 12 months, $n(\%)$ & $798(86)$ & $786(86)$ & $12(63)$ & .014 \\
\hline
\end{tabular}

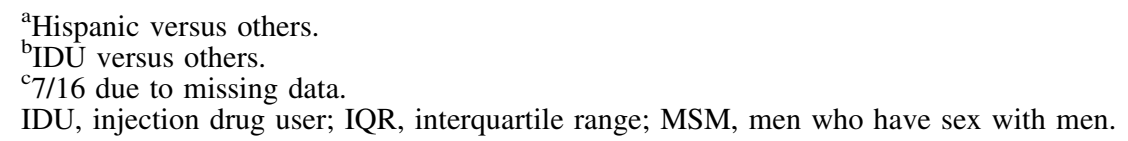

(70\%) (Fig. 1). Five patients with influenza had a CD4 cell count $<200$ cells $/ \mathrm{mm}^{3}$, of whom four contributed more than one specimen for the shedding study.

\section{Influenza shedding study}

Of the 20 persons with influenza, 19 had influenza A (10 with H1N1pdm09, seven with H3N2, and two with subtype unknown) and one had influenza B. Nine (45\%) patients had received pandemic monovalent $\mathrm{H} 1 \mathrm{~N} 1$ vaccine, of whom eight had also received 2010-2011 seasonal trivalent influenza vaccination. Nine patients received oseltamivir within $48 \mathrm{~h}$ of ILI onset. Median days between symptom onset and clinical evaluation differed for patients with and without oseltamivir use ( 2 days vs. 4.5 days, $p=.025$ ). With regard to comorbidities, nine patients had liver disease (six with hepatitis $\mathrm{C}$ and three with hepatitis B), seven had cardiovascular disease, three had a history of asthma, and three had an AIDS-defining cancer.

Median duration of influenza detection by PCR was 10 days (IQR: 6-15 days). Median duration of influenza shedding by viral culture was 3 days (IQR: 0-5 days). No patient shed influenza virus as detected by viral culture for $>7$ days. Median duration of any ILI symptom from ILI onset was 14 days (IQR: 12-26 days). At day 10 after ILI onset, the median RT-PCR Ct value was 37.8; 1 (20\%) specimen had a $\mathrm{Ct}$ value $<30$. The median $\mathrm{Ct}$ values for RT-PCR-positive but culture-negative specimens (based on repeat testing at sequential visits) were 36.2 (IQR: 31.1-38.9) for influenza A, 36.6 (IQR: 31.1-40.6) for influenza B, and 34.0 (IQR: 31.4 36.5 ) for influenza A/H1N1.
FIG. 1. Symptoms reported by HIV-infected persons with influenza $(n=20)$, 2010-2011.

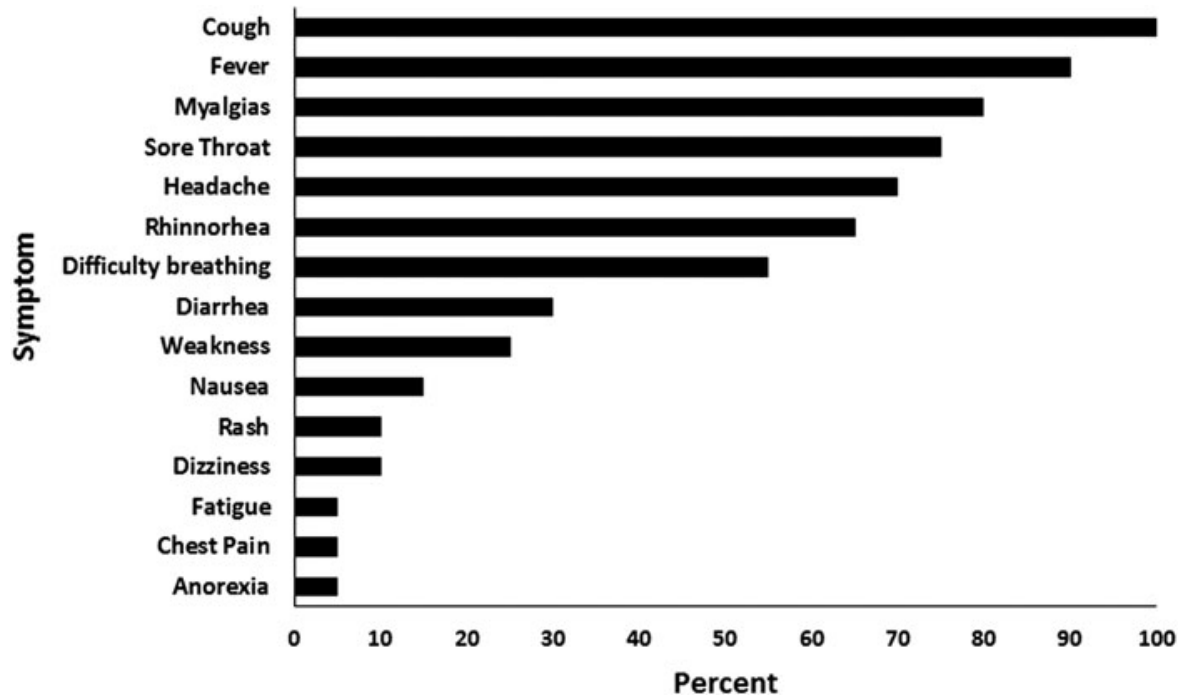


Median days of shedding were similar among patients with and without prior influenza vaccination in the past 12 months ( 0 day vs. 4 days, $p=.448$ ), among patients with and without HIV viral suppression ( 2 days vs. 6 days, $p=.053$ ), and among patients who were eligible for oseltamivir treatment (seen within $72 \mathrm{~h}$ of symptom onset) who did and did not receive oseltamivir ( 5 days vs. 0 days, $p=.083$ ). Those on oseltamivir had similar CD4+ cell counts, 312 cells $/ \mathrm{mm}^{3}$ (176-444) vs. 443 (300-568), $p=.428$. Duration of symptoms were also similar among persons who did and did not receive oseltamivir (13 days vs. 20.5 days, $p=.302$ ). CD4 cell count is correlated with shedding time, $r=-0.481, p=.043$. Only one patient whose CD4 cell count was 176 cells $/ \mathrm{mm}^{3}$ and HIV viral load was 1,025 copies/ml shed viable influenza virus 2 days after his symptoms resolved (total duration of influenza shedding $=6$ days). One patient whose CD4 cell count was 128 cells $/ \mathrm{mm}^{3}$ and HIV RNA was $<20$ copies/ml died 1 day after diagnosis of influenza and pneumonia. There were no hospitalizations. Among ILI symptoms, only a longer duration of fever correlated with a longer duration of influenza virus shedding (Fig. 2).
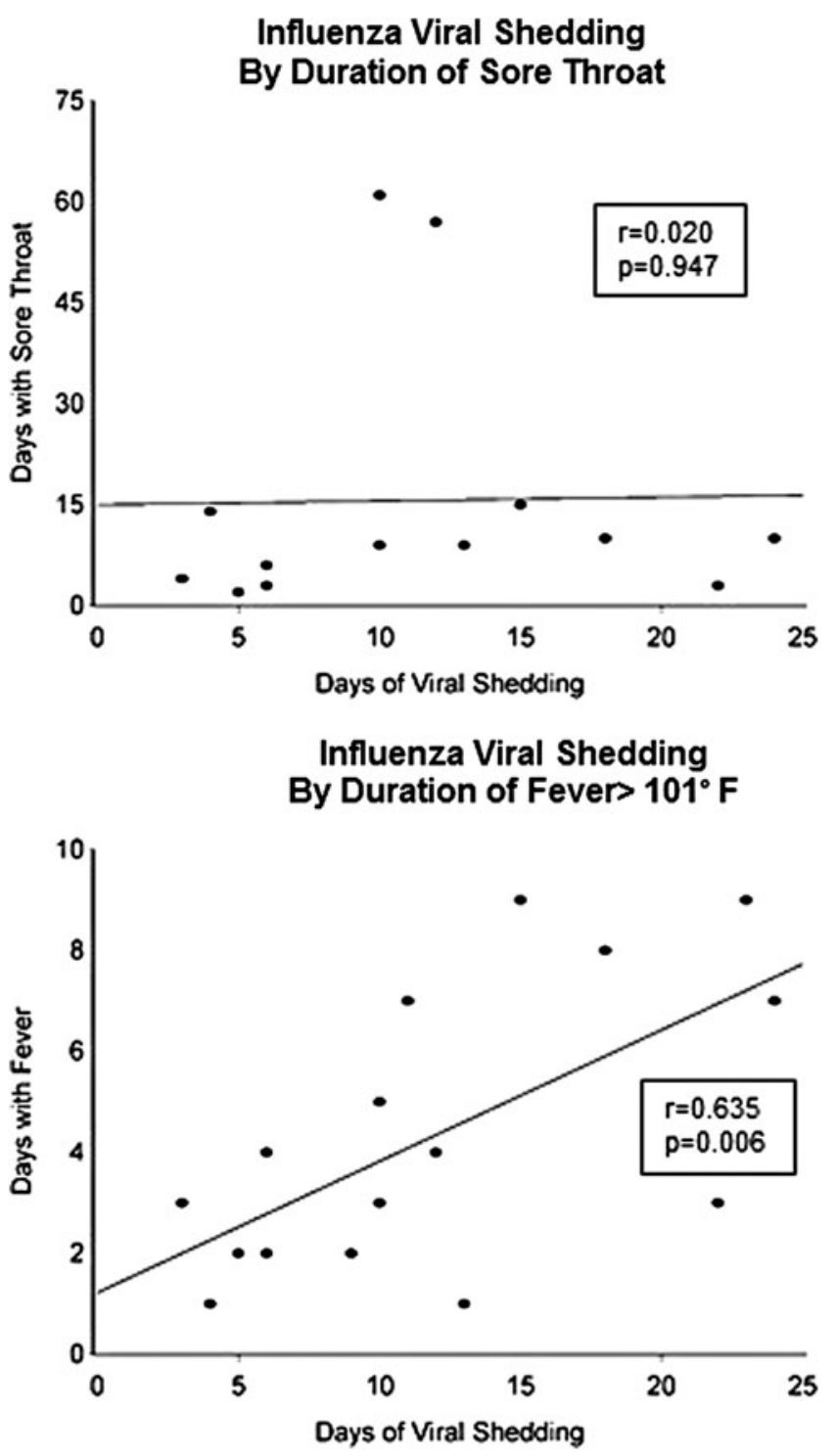

\section{Discussion}

We found that HIV-infected persons shed influenza virus for a similar average duration to that reported for HIVuninfected persons (7-10 days). ${ }^{1-5}$ Furthermore, we found that the median duration of shedding was 10 days for PCR detection and 3 days for viral isolation. These findings are not consistent with previous reports of prolonged shedding of influenza virus among persons immunocompromised by HIV infection or other conditions. ${ }^{13-20} \mathrm{We}$ surmise that the better immune health (i.e., higher CD4 cell counts and mostly suppressed plasma HIV RNA viral loads) of the antiretroviral-treated HIV-infected patients in our study, compared with immunocompromised persons in prior reports, made them less likely to experience prolonged shedding of influenza virus. Only one person in our study died; a participant with a low CD4 cell count $(<200$ cells/ $\mathrm{mm}^{3}$ ), which is a known risk for more severe influenza illness.

In our study, the duration of influenza virus shedding was similar, for persons who received a prior influenza

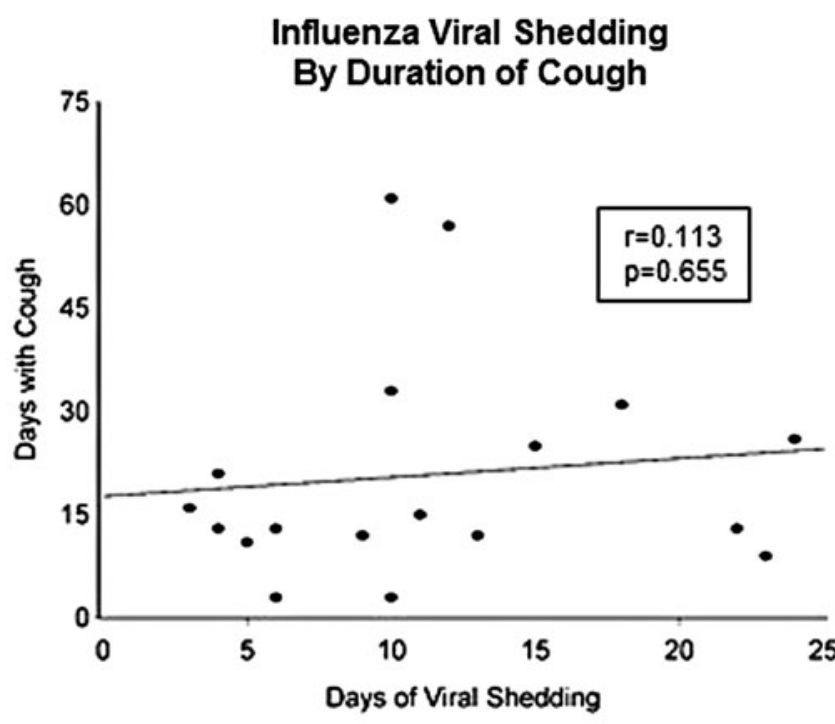

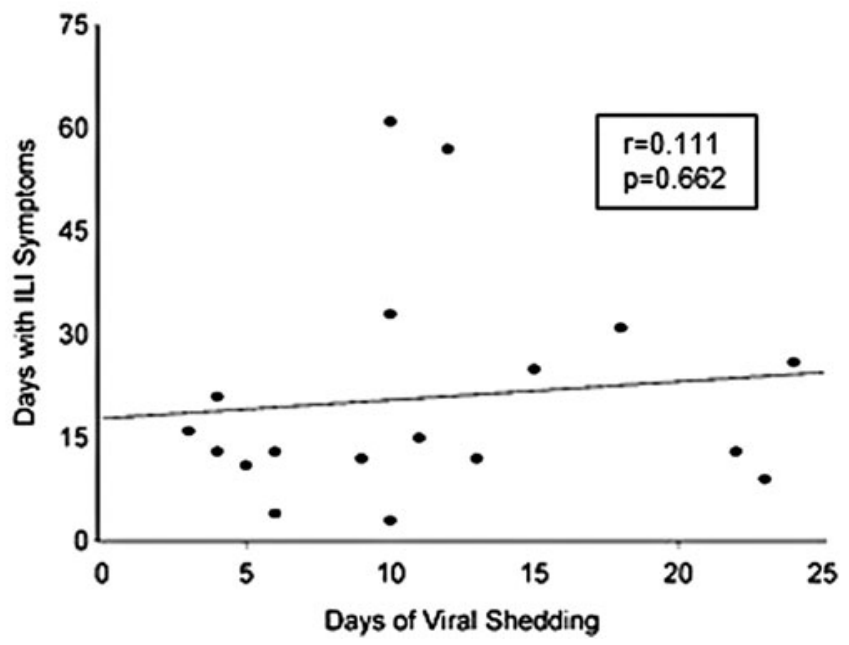

FIG. 2. Correlation of symptoms with influenza virus shedding by PCR positivity, 2010-2011. 
vaccination and for persons whose HIV infection was virologically suppressed in plasma. Persons diagnosed with influenza were more likely than those not diagnosed with influenza to be older, injection drug users, have a low median CD4 cell count, have a detectable HIV viral load, and have no history of having an influenza vaccination in the past 12 months. For HIV-infected patients diagnosed with influenza, early treatment with antivirals targeting influenza may shorten the duration of symptoms and illness ${ }^{26}$ and shorten the duration of influenza virus shedding. ${ }^{27,28}$ The effect of vaccination on virus shedding in HIV-infected persons has not been evaluated before, and our results suggest that this should be looked at more rigorously. Influenza-like symptoms lasted longer than influenza virus shedding among most study participants, and the only symptom that correlated with influenza virus shedding by PCR was fever. Previous studies have reported similar findings, suggesting that fever is closely correlated with viral shedding and that higher fever may reflect higher infectiousness. ${ }^{29,30}$ Clinicians should be aware that persons with influenza and fever are likely to be shedding virus and are infectious. For HIV-infected adults, fever may be a useful indicator for shedding so long as the potentially confounding effect of antipyretic therapy is accounted for. In addition, fever may be a good indication to institute necessary precautions, such as respiratory hygiene and hand washing, to prevent the further spread of influenza.

Our study had limitations. Our sample of persons diagnosed with influenza was small and the 2010-2011 influenza season during which our data were collected was mild. ${ }^{31}$ Our oldest patient was age 53 years and we cannot know if our findings are generalizable to the growing population of elderly persons with HIV infection. We were also unable to adequately assess the duration of shedding among persons with severe (CD4 cell count $<200$ cells $/ \mathrm{mm}^{3}$ ) immunodeficiency since $85 \%$ of participants diagnosed with influenza had a suppressed plasma HIV viral load and 75\% had a CD4 cell count $\geq 200$ cells $/ \mathrm{mm}^{3}$. Our sample size was small, and thus, the study was not powered to assess the efficacy of vaccination or antiviral therapy in HIV-infected persons with influenza. Finally, few participants had comorbidities, and our small sample size limited our capacity to assess the effect of comorbidities on viral shedding.

In conclusion, we found that HIV-infected adults receiving suppressive cART shed influenza for a period similar to that estimated among HIV-uninfected persons with influenza. Fever correlated most closely with viral shedding and infectiousness. Annual influenza vaccination with inactivated formulations is recommended for all HIV-infected adults to prevent illness. Our data suggest HIV viral suppression may attenuate the duration of influenza shedding. Additional efforts are needed to evaluate the effect of vaccination on infectiousness and transmission of influenza virus infection.

\section{Ethical Considerations}

The investigation followed the guidelines of the U.S. Department of Health and Human Services regarding protection of human subjects. The study protocol was approved and renewed annually by each participating institutions' ethical review board. All study participants provided written informed consent.

\section{Financial Support}

Centers for Disease Control and Prevention contract numbers 200-2002-00610, 200-2002-00611, 200-2002-00612, 200-2002-00613, 200-2007-23633, 200-2007-23634, 2002007-23635, and 200-2007-23636.

\section{Disclaimer}

The findings and conclusions from this review are those of the authors and do not necessarily represent the views of the Centers for Disease Control and Prevention.

\section{Author Disclosure Statement}

E.T.O. has served as a consultant or on an advisory board for the following companies: Gilead, Bristol Myers Squibb, GlaxoSmithKline, Tibotec, Merck, and Monogram Sciences. K. H. has received research support from Gilead and GlaxoSmithKline/ViiV.

\section{References}

1. Lee N, Chan PKS, Hui DSC, Rainer TH, Wong E, Choi $\mathrm{KW}$, et al:: Viral loads and duration of viral shedding in adult patients hospitalized with influenza. J Infect Dis 2009;200:492-500.

2. Ryoo SM, Kim WY, Sohn CH, Seo DW, Oh BJ, Lee JH, et al.: Factors promoting the prolonged shedding of the pandemic (H1N1) 2009 influenza virus in patients treated with oseltamivir for 5 days. Influenza Other Respir Viruses 7:833-837.

3. Meschi S, Selleri M, Lalle E, Bordi L, Valli MB, Ferraro F, et al:: Duration of viral shedding in hospitalized patients infected with pandemic H1N1. BMC Infect Dis 2011;11:140.

4. Na S, Chong YP, Kim MN, Kim WY, Kim W, Hong SB, et al:: Duration of viral shedding in patients admitted to hospital with pandemic influenza A/H1N1 2009 infection. J Med Virol 2011;83:5-9.

5. Waiboci LW, Lebo E, Williamson JM, Mwiti W, Kikwai GK, Njuguna $\mathrm{H}$, et al.: Viral shedding in patients infected with pandemic influenza A (H1N1) virus in Kenya, 2009. PLoS One 2011;6:e20320.

6. Klein MB, Lu Y, DelBaso L, Cote S, Boivin G: Influenza virus infection is a primary cause of febrile respiratory illness in HIV-infected adults, despite vaccination. Clin Infect Dis 2007;45:234-240.

7. Cohen JP, Macauley C: Susceptibility to influenza A in HIV-positive patients. JAMA 1989;261:245.

8. Thurn JR, Henry K: Influenza A pneumonitis in a patient infected with human immunodeficiency virus (HIV). Chest 1989;95:807-810.

9. Safrin S, Rush JD, Mills J: Influenza in patients with human immunodeficiency virus infection. Chest 1990;98:33-37.

10. Radwan HM, Cheesman SH, Lai KK, Ellison IR: Influenza in human immunodeficiency virus-infected patients during the 1997-1998 influenza season. Clin Infect Dis 2000;31:604-606.

11. Neuzil KM, Reed GW, Mitchel EF Jr, Griffen MR: Influenza-associated morbidity and mortality in young and middle-aged women. JAMA 1999;281:901-907.

12. Lin JC, Nichol KL: Excess mortality due to pneumonia or influenza during influenza seasons among persons with acquired immunodeficiency syndrome. Arch Intern Med 2001;161:441-446.

13. Baz M, Abed Y, McDonald J, Boivin G: Characterization of multidrug-resistant influenza A/H3N2 viruses shed dur- 
ing 1 year by an immunocompromised child. Clin Infect Dis 2006;43:1555-1561.

14. Boivin G, Goyette N, Bernatchez H: Prolonged excretion of amantadine-resistant influenza A virus quasi species after cessation of antiviral therapy in an immunocompromised patient. Clin Infec Dis 2002;34:e23-e25.

15. Gooskens J, Jonges M, Claas EC, Meijer A, Kroes ACM: Prolonged influenza virus infection during lymphocytopenia and frequent detection of drug-resistant viruses. J Infect Dis 2009; 199:1435-1441.

16. Klimov AI, Rocha E, Hayden FG, Shult PA, Roumillat LF, Cox NJ: Prolonged shedding of amantadine-resistant influenza A viruses by immunodeficient patients: Detection by polymerase chain reaction-restriction analysis. J Infect Dis 1995; 172:1352-1355.

17. Weinstock DM, Gubareva LV, Zuccotti G: Prolonged shedding of multidrug-resistant influenza virus in an immunocompromised patient. N Engl J Med 2003;348:867-868.

18. Ison MG, Gubareva LV, Atmar RL, Treanor J, Hayden FG: Recovery of drug-resistant influenza virus from immunocompromised patients: A case series. J Infect Dis 2006;193: 760-764.

19. van der Vries E, van den Berg B, Scutten M: Fatal Oseltamivir-resistant influenza virus infection. N Engl J Med 2008;359:1074-1076.

20. Pemberton RM, Jennings R, Potter CW, Oxford JS: Amantadine resistance in clinical influenza A (H3N2) and (H1N1) virus isolates. J Antimicrob Chemoth 1986;18: 135-140.

21. Le QM, Kiso M, Someya K, et al.: Avian flu: Isolation of drug-resistant H5N1 virus. Nature 2005;437:1108.

22. Herlocher ML, Truscon R, Elias S, et al:: Influenza viruses resistant to the antiviral drug oseltamivir: Transmission studies in ferrets. J Infect Dis 2004;190:1627-1630.

23. CDC: Monitoring selected national HIV prevention and care objectives by using HIV surveillance data-United States and 6 dependent areas-2012. HIV Surveillance Supplemental Report 2014;19 (No. 3). Published November 2014.
24. Vellozzi C, Brooks JT, Bush T, et al:: The study to understand the natural history of HIV and AIDS in the era of effective therapy (SUN study). Am J Epidemiol 2009;169: 642-652.

25. Hilton JF: The appropriateness of the Wilcoxon test in ordinal data. Stat Med 1996;15:631-645.

26. Fiore AE, Fry A, Shay D, et al.: Antiviral agents for the treatment and chemoprophylaxis of influenza-Recommendations of the Advisory Committee on Immunization Practices (ACIP). MMWR Recomm Rep 2011;60:1-24.

27. Ling LM, Chow AL, Lye DC, Tan AS, Krishnan P, Cui L, et al.: Effects of early oseltamivir therapy on viral shedding in 2009 pandemic influenza A (H1N1) virus infection. Clin Infect Dis 2010;50:963-969.

28. Leung YH, Lim WL, Wong MH, Chuang SK: Delayed oseltamivir treatment is associated with longer viral shedding of pandemic (H1N1) 2009 virus. Epidemiol Infect 2012;140:814-817.

29. Hayden FG, Fritz R, Lobo MC, Alvord W, Strober W, Straus SE: Local and systemic cytokine responses during experimental human influenza A virus infection: Relation to symptom formation and host defense. J Clin Invest 1998;101:643-649.

30. Lau LL, Cowling BJ, Fang VJ, Kwok-Hung C, Lau EHY, Lipsitch $\mathrm{M}$, et al.: Viral shedding and clinical illness in naturally acquired influenza virus infections. J Infect Dis 2010;201:1509-1516.

31. Centers for Disease Control and Prevention: Update: Influenza activity-United States, 2010-2011 season, and composition of the 2011-2012 influenza vaccine. MMWR Morb Mortal Wkly Rep 2011;60:705-712.

Address correspondence to:

Pragna Patel

Centers for Disease Control and Prevention 1600 Clifton Road Atlanta, GA 30333

E-mail: plp3@cdc.gov 